\title{
1. Wirtschaftliche Entwicklung 1996 nach Regionen
}

\section{(2) OpenEdition}

\section{Journals}

Electronic version

URL: http://journals.openedition.org/sjep/696

DOI: $10.4000 /$ sjep.696

ISSN: 1663-9677

\section{Publisher}

Institut de hautes études internationales et du développement

\section{Printed version}

Date of publication: 1 avril 1998

Number of pages: 129-135

ISSN: $1660-5926$

\section{Electronic reference}

«1. Wirtschaftliche Entwicklung 1996 nach Regionen », Schweizerisches Jahrbuch für Entwicklungspolitik [Online], 17 | 1998, Online erschienen am: 03 August 2012, abgerufen am 08 September 2020. URL : http://journals.openedition.org/sjep/696 ; DOI : https://doi.org/10.4000/sjep. 696 


\section{WIRTSCHAFTLICHE ENTWICKLUNG 1996 NACH REGIONEN}

D

IESES KAPITEL gibt einen kurzen Überblick über die wirtschaftliche Entwicklung im Jahr 1996, nach grossen geographischen Regionen aufgegliedert. Ferner werden die Beziehungen der Schweiz mit den südlichen und den mittel- und osteuropäischen Entwicklungsländern im Rahmen ihrer wirtschaftlichen Entwicklung erläutert. Die hier präsentierten Daten sind den Jahresberichten der wichtigsten internationalen Wirtschaftsorganisationen (Internationaler Währungsfonds, Weltbank, Bank für Internationalen Zahlungsausgleich, Organisation für wirtschaftliche Zusammenarbeit und Entwicklung) entnommen.

1996 verzeichnete die Weltwirtschaft im dritten aufeinanderfolgenden Jahr ein Wachstum von rund 4\% (Tabelle Nr. 1). In vielen Industrieländern hat sich die Wirtschaftstätigkeit wieder erholt, aber je nach den Ländern der Zone in unterschiedlicher Weise. Die Entwicklungsländer hielten ihre Wachstumsrate auf über $6 \%$ (vgl. Tabelle Nr. 2). Die lateinamerikanischen Länder, die von der Finanzkrise Mexikos 1995 betroffen worden waren, befinden sich wieder auf Expansionskurs. Auch im Mittleren Osten und in noch ausgeprägterem Masse in Afrika hat sich das Wirtschaftswachstum beschleunigt. In den Transitionsländern hat sich die Produktion nach sechs Jahren starker Schrumpfung global stabilisiert. Schliesslich wurde in mehreren aufstrebenden Volkswirtschaften Asiens ein Nachlassen des Wachstums verzeichnet (Tabelle Nr. 3) '.

Tabelle Nr. 1

Jährliche Wachstumsrate des Bruttosozialprodukts (in \%)

\begin{tabular}{lrrrr}
\hline & $\mathbf{1 9 9 3}$ & $\mathbf{1 9 9 4}$ & $\mathbf{1 9 9 5}$ & $\mathbf{1 9 9 6}$ \\
\hline Weltweit & $\mathbf{2 . 7}$ & $\mathbf{4 . 1}$ & $\mathbf{3 . 7}$ & $\mathbf{4 . 0}$ \\
\hline Industrieländer & 1.2 & 3.1 & 2.5 & 2.5 \\
\hline Entwicklungsländer & 6.5 & 6.8 & 6.0 & 6.5 \\
\hline Transitionsländer* & -6.5 & -6.7 & -0.8 & -0.1 \\
\hline
\end{tabular}

* Ex-UdSSR, Bulgarien, Tschechien, Slowakei, Rumänien, Polen, Ungarn

Quelle: IWF-Jahresbericht 1997

\subsection{INDUSTRIELÄNDER ${ }^{2}$}

1996 erreichte das Wirtschaftswachstum in den OECD-Ländern 2,5\%, was in etwa der Rate von 1995 entspricht, jedoch war seine Entwicklung recht unterschiedlich. Die stärksten Impulse gingen von der amerikanischen und der britischen Wirtschaft aus. Die Inlandsnachfrage in beiden Ländern, besonders der

1. Internationaler Währungsfonds, Jahresbericht 1997, Washington, IWF, April 1997.

2. Schweizerische Nationalbank, 89e rapport de gestion 1996, Zürich, SNB, 1997, S. 7. 
Konsum, stieg an, jedoch haben sich die Ausfuhren ungleich entwickelt. Die Festigkeit des Dollars und der Nachfragerückgang in Europa haben die amerikanischen Exporte gebremst, während der Auslandsabsatz Grossbritanniens noch zunahm. Im zweiten Halbjahr begann der europäische Kontinent dank einer festeren Auslandsnachfrage (Frankreich und Deutschland) die Schwächephase zu überwinden, die er seit dem Herbst 1995 verzeichnet hatte.

Das Bruttoinlandsprodukt Japans stieg 1996 nach mehrjähriger Stagnation merklich an. Diese Zunahme erklärt sich vor allem durch die grossen Investitionen der öffentlichen Körperschaften. Trotz niedriger Zinssätze hat die Nachfrage des Privatsektors keinen wirklichen Aufschwung genommen, wahrscheinlich zum Teil wegen der Krise des japanischen Bankensystems. Das Wachstum der Ausfuhren ist infolge der Erstarkung des Yen zwischen Anfang 1993 und Mitte 1995 nahezu zum Stillstand gekommen. Die Einfuhren haben sich ihrerseits kräftig erhöht.

Das feste Wirtschaftswachstum in den USA und Grossbritannien hatte eine Verbesserung der Beschäftigungslage und einen Rückgang der Arbeitslosigkeit zur Folge. Hingegen hat die Arbeitslosigkeit in zahlreichen europäischen Ländern, namentlich in Deutschland und Frankreich, noch zugenommen. Im Durchschnitt der EU-Staaten lag die Arbeitslosenrate unverändert bei rund $11 \%$.

\section{$\square$ Die Schweizer Wirtschaft ${ }^{3}$}

Entgegen den 1995 ergangenen Vorhersagen hatte die schweizerische Volkswirtschaft 1996 wiederum eine leichte Rezession zu verzeichnen. Von einer kurzen Erholung im Jahre 1994 abgesehen, befindet sich die Wirtschaft der Schweiz somit im sechsten aufeinanderfolgenden Jahr in einer Stagnationsphase. Verschiedene Gründe erklären diese unter den westlichen Industrieländern fast einzigartige Situation. Zum einen spielt die starke Höherbewertung des Schweizer Frankens seit 1992, welche bis Mitte 1996 angehalten hat, eine wesentliche Rolle. Zum anderen befindet sich die Schweizer Wirtschaft in einer intensiven Anpassungsphase im Rahmen der wirtschaftlichen Globalisierung. Die Schweiz muss sich an eine immer stärkere internationale Arbeitsteilung und einen erhöhten weltweiten Wettbewerb zwischen den verschiedenen Produktionsstandorten anpassen. Sie muss zudem eine Überkapazität im Bausektor und vor allem strukturelle Defizite in den öffentlichen Finanzen bewältigen. Diese verschiedenen Faktoren bleiben nicht ohne Auswirkungen auf die Beschäftigung (massiver und zum Teil irreversibler Anstieg der Arbeitslosigkeit) und auf den Konsum der privaten Haushalte (in ihrem Ausmass wie in ihrer Hartnäckigkeit einmalige Konsumverschlechterung). Die Hauptkomponente der Inlandsnachfrage, nämlich der private Konsum, wird dadurch ernsthaft beeinträchtigt. Die Investitionen der Unternehmen, die 1994 und 1995 stark gestiegen waren, beschränken sich weiterhin auf Anstrengungen zur Erhaltung der Wettbewerbsfähigkeit bestehender Produktionsstrukturen.

Positive Impulse können somit nur von der Aussenwirtschaft erwartet werden. Jedoch wurde 1996 ein leicht negativer oder bestenfalls neutraler Beitrag der Aussenwirtschaft verzeichnet. Die schweizerischen Ausfuhren litten unter der sehr mässigen Konjunktur in Westeuropa, welches zu ihren wichtigsten Abnehmern zählt, und unter den Folgen der Frankenhausse, die bedeutende Marktanteilsverluste im Ausland und in bestimmten Wirtschaftszweigen wie dem Tou-

3. Bericht zur Aussenwirtschaftspolitik 96/1 + 2, 15. Januar 1996 (Botschaft Nr. 97.002), S. $29 \mathrm{ff}$. 
rismus mit sich brachte. Angesichts der Stärke des Schweizer Frankens haben einige Exportindustrien, hauptsächlich pharmazeutische Produkte, Investitionsgüter und Nahrungsmittel, 1996 gute Leistungen mit einer Steigerung des Exportvolumens um 2,4\% erbracht.

1997 hat sich die Wirtschaftslage etwas verbessert. Gemäss der im Dezember 1997 veröffentlichten Statistik des Bundesamtes für Konjunkturfragen ist das reale Bruttoinlandsprodukt im dritten Quartal gegenüber dem zweiten Quartal um 1,7\% gestiegen. Für 1997 beträgt das Wachstum 0,8\%. Das Bundesamt für Konjunkturfragen anerkennt, dass der Wachstumsrhythmus weiterhin mässig ist, betont jedoch, dass das BIP erstmals seit zwei Jahren eine solche vierteljährliche Wachstumsrate über zwölf Monate hinweg erreichte. Dynamisiert durch einen wesentlich günstigeren Kurs des Schweizer Frankens als 1995 - 1996 und durch die Wiederbelebung der Auslandsnachfrage in Europa sind die schweizerischen Ausfuhren unbestreitbar der Hauptmotor der wirtschaftlichen Erholung ${ }^{4}$.

\subsection{ENTWICKLUNGSLÄNDER}

In den aufstrebenden Volkswirtschaften war das Wirtschaftswachstum im letzten Jahr weiterhin kräftig, und die Inflation ging noch zurück (Tabelle 3). In Lateinamerika hat sich die durchschnittliche Wachstumsrate dank der Erholung in Argentinien und Mexiko erhöht. Afrika hat als Ergebnis der Stabilisierungs- und Reformanstrengungen (gemäss IWF), einer Verbesserung der Rohstoffpreise und einer Zunahme der Investitionen seinen stärksten Wirtschaftsaufschwung seit zwei Jahrzehnten verzeichnet. In Asien haben mehrere Länder restriktive Politiken eingeführt, um einer einsetzenden Konjunkturüberhitzung entgegenzuwirken. Die Lethargie der Exportmärkte hat zum Nachlassen der Wirtschaftstätigkeit in dieser Region im Vergleich zu ihrer früheren Dynamik geführt und eine signifikante Verringerung der grossen externen Ungleichgewichte verhindert. In einer Reihe osteuropäischer Länder, wo der Übergangsprozess bereits fortgeschritten ist, blieb das Wachstum weiterhin anhaltend. In Russland muss sich die Wiederankurbelung der Produktion indessen noch bestätigen, doch setzte sich der tendenzielle Inflationsrückgang fort.

Tabelle Nr. 2

Jährliche Wachstumsrate der Entwicklungsländer (BSP in \%)

\begin{tabular}{lrrrr}
\hline & $\mathbf{1 9 9 3}$ & $\mathbf{1 9 9 4}$ & $\mathbf{1 9 9 5}$ & $\mathbf{1 9 9 6}$ \\
\hline Insgesamt & $\mathbf{6 . 5}$ & $\mathbf{6 . 8}$ & $\mathbf{6 . 0}$ & $\mathbf{6 . 5}$ \\
\hline Afrika & 0.9 & 2.9 & 2.9 & 5.1 \\
\hline Asien & 9.3 & 9.6 & 8.9 & 8.2 \\
\hline Mittlerer Osten" & 4.3 & 0.3 & 3.8 & 4.5 \\
\hline Lateinamerika und Karibik & 3.7 & 5.0 & 1.3 & 3.5 \\
\hline
\end{tabular}

Quelle: IWF-Jahresbericht 1997.

* einschliesslich Malta, Türkei und Zypern. Anmerkung: Die Zahlen für das Wachstum nach grossen Regionen stimmen mit denen des Berichts der BIZ nicht überein. Dies erklärt sich durch die verschiedenen statistischen Methoden der beiden Finanzinstitute.

4. In Journal de Genève, 11. Dezember 1997 «Dopée par les exportations, mais pénalisée par la construction, l'économie suisse croît modérément ». 


\section{$\square$ Asien}

Unter dem Einfluss restriktiver Geldpolitiken und eines Rückgangs des Exportwachstums hat die Zunahme des Produktionsvolumens der dynamischen Volkswirtschaften Asiens ${ }^{5} 1996$ etwas nachgelassen und wird auf 6\% geschätzt. Der Exportrückgang war zum Teil auf die schwache Nachfrage im OECD-Raum und auf den regionalen Märkten und zum Teil auf die negativen Auswirkungen der Höherbewertung des Dollars auf die Wettbewerbsfähigkeit zurückzuführen, da viele Währungen der Region an den Dollar gebunden sind. Parallel dazu ging die Inflation geringfügig zurück und erreichte 4,5\%. Das Nachlassen der Wirtschaftstätigkeit war besonders markant in Thailand, wo die Inlandsnachfrage vor allem unter den Auswirkungen der Baisse der Immobilienbörsenmärkte auf die Bilanzen und unter dem Vertrauensschwund gelitten hat. In Taiwan und Hongkong war der Rückgang, namentlich dank des anhaltenden Wachstums in China, vergleichsweise weniger stark ausgeprägt.

In China hat der Nachfragedruck noch weiter nachgelassen. Die restriktive Politik und die strenge Kontrolle der neuen Investitionsprojekte der öffentlichen Unternehmen haben weitgehend zu dieser Entwicklung beigetragen. Der beträchtliche Rückgang des Exportwachstums, das von rund 25\% (in Dollar) im Jahr 1995 auf unter 5\% 1996 gesunken ist, hat ebenfalls eine mässigende Rolle gespielt. Der Anstieg der Einzelhandelspreise ging von einer Jahresrate von $15 \%$ 1995 auf 5\% 1996 zurück. Das Wachstum des realen BIP sank leicht ab auf $10 \%$, wobei die relativ gute Leistung des Agrarsektors einen markanteren Rückgang der Gesamtproduktion verhinderte. Seit Ende 1996 hat sich das Exportwachstum merklich beschleunigt. Die Ausfuhren in die USA, die rund $18 \%$ der chinesischen Gesamtausfuhren ausmachen, waren besonders kräftig .

In Indien haben die seit der Öffnung des Handelsaustausches 1991 erzielten Fortschritte, die Liberalisierung des Finanzsektors und die Haushaltsreform zur Beschleunigung des Wachstums beigetragen, das im Verlauf der letzten fünf Jahre durchschnittlich $6 \%$ erreicht hat. Gleichzeitig wurde die Inflation gesenkt ${ }^{7}$.

Dahrbuch 1998, Internationale Finanzbeziehungen (p. 137)

\section{$\square$ Lateinamerika}

Die Wachstumsrate für Lateinamerika stieg von 0,5\% 1995 auf 3,5\% 1996 an. Die Erhöhung der durchschnittlichen Wachstumsrate erklärt sich hauptsächlich durch den Wiederaufschwung in Argentinien und Mexiko. Parallel dazu haben verschiedene Inflationsbekämpfungsstrategien in den letzten Jahren ihre Ergebnisse gezeitigt, die durchschnittliche Inflationsrate liegt 1996 bei $25 \%$. Sie betrug 1995 43\%, was bereits einem guten Resultat gleichkam, denn die durchschnittliche Inflationsrate im Zeitraum 1990 - 1997 lag bei $257 \%{ }^{8}$.

Die Erholung der Wirtschaftstätigkeit, die Mitte 1996 einsetzte, verstärkt sich weiterhin, vor allem in Argentinien, Brasilien und Peru. In diesen drei Ländern haben der Anstieg der Kapitalzuflüsse und die Entspannung der internen Nominalzinssätze die Voraussetzungen für einen allgemeinen Wiederaufschwung

5. Taiwan, Hongkong, Malaysia, die Philippinen, Singapur und Thailand.

6. OECD, Perspectives économiques de l'OCDE 1997, Paris, OECD, Juni 1997, S. 133 - 134.

7. Bank für Internationalen Zahlungsausgleich, 67. Jahresbericht, Basel, BIZ, Juni 1997.

8. Bank für Internationalen Zahlungsausgleich, 67. Jahresbericht, Basel, BIZ, Juni 1997. 
geschaffen. Das Wachstum des privaten Verbrauchs und der festen Investitionen war besonders anhaltend. Der in voller Expansion befindliche Erdölsektor hat auch in Venezuela, nach dem plötzlichen Rückgang der Produktion im Zusammenhang mit dem Stabilisierungsplan von Anfang 1996, einen Wiederaufschwung ausgelöst. Ausser in Argentinien bleibt die Inflation hoch, geht aber unter dem Einfluss hoher kurzfristiger Realzinssätze, hoher Arbeitslosenraten und einer leichten Erhöhung des realen Wechselkurses weiter zurück. In Chile und Kolumbien hat die Wirtschaftstätigkeit aufgrund der strikten Geldpolitiken 1996 nachgelassen. Die Inflation in Chile weist erneut eine fallende Tendenz auf, was den Ansatz einer allmählichen Senkung der Zinssätze erlaubte. Die Leistungsbilanzdefizite haben sich 1996 im grössten Teil der Region noch erhöht, da der Auslandsschuldendienst gestiegen ist ; die Einfuhrnachfrage hat zugenommen, und die Austauschverhältnisse haben sich in den meisten Ländern, mit Ausnahme Venezuelas, verschlechtert ${ }^{9}$.

\section{$\square$ Afrika}

In Afrika hat sich die 1994 und 1995 eingeleitete wirtschaftliche Erholung 1996 noch verstärkt (Tabelle 2 und 3). Der 1996 verzeichnete Konjunkturanstieg von $5 \%$ stellt in der Tat die beste Leistung Afrikas der letzten zwanzig Jahre dar. Darüber hinaus übertraf die Wachstumsrate erstmals den Anstieg des Bevölkerungswachstums. Diese Wachstumsbeschleunigung zeugt von der Dynamik des Rohstoffsektors und in manchen Fällen auch des Verarbeitungssektors. Die Länder der Franc-Zone haben nach der Anpassung des CFA-Francs 1994 einen anhaltenden Wiederaufschwung verzeichnet. Äthiopien, Malawi, Marokko und Tunesien gehören ebenfalls zu den Ländern, die eine kräftige Expansion aufwiesen. Hingegen wurde das Wachstum Südafrikas durch den unerlässlichen Abbau der makroökonomischen Ungleichgewichte und die strukturelle Starrheit weiterhin gebremst ${ }^{10}$. Die UNCTAD stellt fest, dass der in den afrikanischen Ländern südlich der Sahara beobachtete umfassende Wiederaufschwung hauptsächlich auf eine bessere Führung der öffentlichen Geschäfte, auf die festen Preise für bestimmte Rohstoffe sowie auf merklich mildere Wetterbedingungen und einen Rückgang der bürgerlichen Unruhen zurückzuführen ist ${ }^{11}$.

\subsection{TRANSITIONSLÄNDER}

In den Volkswirtschaften Mittel- und Osteuropas setzt sich der Übergangsprozess fort. Die Prekarität des Wachstums ist jedoch offensichtlich und in einigen Fällen beunruhigend geworden. Zahlreiche Staaten der Region sind weitgehend vom Exportaufschwung abhängig und haben daher 1996 unter der Flaute in Westeuropa gelitten. Probleme gibt es auch beim Angebot, das Schwierigkeiten bei der Verbesserung der Qualität und Diversität der Produkte widerspiegelt. Die Arbeitslosenraten sind weiter zurückgegangen, doch drückt die Langsamkeit dieses Rückgangs strukturelle Mängel und insbesondere regionale Unterschiede aus.

9. OECD, Perspectives économiques de l'OCDE 1997, Paris, OECD, Juni 1997, S. 136.

10. Internationaler Währungsfonds, Jahresbericht 1997, Washington, IWF, April 1997.

11. Conférence des Nations Unies sur le commerce et le développement, Rapport sur le commerce et le développement 1997, Genf, UNCTAD, 1997. 
Tabelle Nr. 3

Entwicklung des BIP und der Verbraucherpreise nach Regionen

\begin{tabular}{|c|c|c|c|c|c|c|}
\hline \multirow{3}{*}{ Land/Region } & \multicolumn{3}{|c|}{ BIP real } & \multicolumn{3}{|c|}{ Verbraucherpreise $^{1}$} \\
\hline & \multicolumn{5}{|c|}{ Jährliche Veränderung in \% } & \multirow[b]{2}{*}{1996} \\
\hline & 1990-94 & 1995 & 1996 & $1990-94$ & 1995 & \\
\hline & \multicolumn{2}{|c|}{ Durchschnitt } & \multicolumn{3}{|c|}{ Durchschnitt } & \\
\hline China & 10.5 & 10.2 & 9.7 & 11.6 & 16.8 & 8.3 \\
\hline Indien & 4.6 & 7.1 & 6.8 & $10.5^{1}$ & $9.3^{1}$ & $5.9^{1}$ \\
\hline Übriges Asien² & 7.0 & 7.8 & 6.8 & 6.9 & 6.4 & 5.9 \\
\hline Korea & 7.6 & 8.9 & 7.1 & 7.0 & 4.5 & 5.0 \\
\hline Hongkong & 5.2 & 4.7 & 4.7 & 9.5 & 9.2 & 6.3 \\
\hline Singapur & 8.6 & 8.8 & 7.0 & 2.9 & 1.7 & 1.4 \\
\hline Taiwan & 6.5 & 6.1 & 5.7 & 3.8 & 3.7 & 3.1 \\
\hline Indonesien & 6.9 & 8.5 & 7.5 & 8.6 & 9.4 & 7.9 \\
\hline Malaysia & 8.7 & 9.5 & 8.2 & 3.8 & 3.4 & 3.5 \\
\hline Philippinen & 1.9 & 4.8 & 5.5 & 11.6 & 8.1 & 8.4 \\
\hline Thailand & 9.0 & 8.6 & 6.7 & 4.8 & 5.8 & 5.8 \\
\hline Lateinamerika $^{2}$ & 3.1 & 0.5 & 3.5 & 256.8 & 42.5 & 24.7 \\
\hline Argentinien & 6.8 & -4.6 & 4.4 & 148.3 & 3.4 & 0.2 \\
\hline Brasilien & 0.9 & 4.2 & 2.9 & 1425.9 & 66.0 & 15.5 \\
\hline Chile & 6.4 & 8.5 & 7.2 & 17.4 & 8.2 & 7.4 \\
\hline Kolombien & 4.3 & 5.2 & 2.1 & 26.3 & 20.9 & 20.9 \\
\hline Mexiko & 3.9 & -6.2 & 5.1 & 16.1 & 35.0 & 34.4 \\
\hline Venezuela & 3.9 & 3.4 & -1.6 & 40.7 & 59.9 & 99.9 \\
\hline Osteuropa $^{2}$ & -2.5 & 5.5 & 4.7 & 62.1 & 23.2 & 17.8 \\
\hline Ungarn & -3.3 & 1.5 & 1.0 & 25.4 & 28.3 & 23.6 \\
\hline Polen & -1.6 & 7.0 & 6.0 & 97.6 & 27.7 & 19.9 \\
\hline Tschechische Republik ${ }^{3}$ & -4.2 & 4.8 & 4.4 & 20.5 & 9.1 & 8.8 \\
\hline Russland $^{4}$ & -8.7 & -4.2 & -6.0 & 347.0 & 197.5 & 47.7 \\
\hline Saudiarabien & 4.1 & 1.6 & 2.4 & 1.7 & 4.9 & 1.2 \\
\hline Israel & 5.8 & 7.1 & 4.4 & 14.2 & 10.0 & 11.3 \\
\hline Afrika & 1.4 & 2.7 & 5.1 & 37.2 & 38.4 & 25.0 \\
\hline Südafrika & 0.1 & 3.3 & 3.1 & 12.4 & 8.6 & 7.4 \\
\hline
\end{tabular}

Daten für 1996 teilweise geschätzt.

1. Grosshandelspreise.

2. Gewogener Durchschnitt der aufgeführten Länder auf der Basis des BIP und der Kaufkraftparitäten von 1990.

3. Vor 1985: Tschechoslowakei.

4. Vor 1986: Sowjetunion.

Quelle: Bank für Internationalen Zahlungsausgleich, 67. Jahresbericht, Juni 1997. 
In Russland war ein starker Rückgang der Inflationsrate festzustellen, was ein Wiederanlaufen der Produktion erlauben dürfte. In Polen, wo die Expansionsphase ins fünfte Jahr ging, hat das Wachstum etwas nachgelassen und lag bei $5,5 \%$. In Ungarn hat sich das Wachstum verlangsamt und erreichte $1 \%$. Hingegen zeigt die Entwicklung in Bulgarien und Rumänien, dass die Transitionspolitiken keinen genügenden Strukturwandel ermöglichen, was gemäss der OECD der Glaubwürdigkeit der globalen Geld- und Wirtschaftspolitiken schaden kann. Die allgemeinen Aussichten bezüglich des Wirtschaftswachstums und der Handelsströme scheinen für 1997 zufriedenstellender zu sein, jedoch bleibt die Umstrukturierung von der Planwirtschaft zur Marktwirtschaft eine komplexe Aufgabe ${ }^{12}$.

\section{QUELLEN}

Bericht zur Aussenwirtschaftspolitik 96/1 + 2, 15. Januar 1997 (Botschaft Nr. 97.002). Bank für Internationalen Zahlungsausgleich, 67. Jahresbericht, Basel, BIZ, Juni 1997.

OECD, Perspectives économiques de l'OCDE 1997, Paris, OECD, Juni 1997.

Internationaler Währungsfonds, Jahresbericht 1997, Washington, IWF, April 1997.

Schweizerische Nationalbank, 89e rapport de gestion 1996, Zürich, SNB, 1997.

Weltbank, L'Etat dans un monde en mutation, Washington, Weltbank, Juni 1997.

United Nations Conference on Trade and Development, Trade and Development Report 1997, New York and Geneva, UNCTAD, 1997.

United Nations Conference on Trade and Development, The Least Developed Countries 1997 Report, New York and Geneva, UNCTAD, 1997.

\section{INTERNET-ADRESSEN}

Weltbank: http://www. worldbank. org/

Internationaler Währungsfonds: http://www. imf. org/

OECD : http://www. OECD. org/

UNCTAD: http://www. unicc. org/unctad/ 